\title{
Teaching object-oriented programming on top of functional programming
}

\author{
Kristensen, Jens Thyge; Hansen, Michael Reichhardt; Richel, Hans
}

Published in:

Proceedings on 31st Annual Frontiers in Education Conference

Link to article, DOI:

10.1109/FIE.2001.963848

Publication date:

2001

Document Version

Publisher's PDF, also known as Version of record

Link back to DTU Orbit

Citation $(A P A)$ :

Kristensen, J. T., Hansen, M. R., \& Richel, H. (2001). Teaching object-oriented programming on top of functional programming. In Proceedings on 31st Annual Frontiers in Education Conference (Vol. 1, pp. 15-20). IEEE. https://doi.org/10.1109/FIE.2001.963848

\section{General rights}

Copyright and moral rights for the publications made accessible in the public portal are retained by the authors and/or other copyright owners and it is a condition of accessing publications that users recognise and abide by the legal requirements associated with these rights.

- Users may download and print one copy of any publication from the public portal for the purpose of private study or research.

- You may not further distribute the material or use it for any profit-making activity or commercial gain

- You may freely distribute the URL identifying the publication in the public portal 
Session T1D

\title{
Teaching Object-Oriented Programming on Top of Functional Programming
}

\author{
Jens Thyge Kristensen, Michael R. Hansen and Hans Rischel ${ }^{1}$
}

\begin{abstract}
In the Informatics Programme at the Technical University of Denmark, we base the first course in objectoriented programming (using the Java language) on a preceding course in functional programming (using the SML language). The students may hence exploit concepts from functional programming in the construction of OO programs. This is done following a method where the program design is expressed in SML and afterwards implemented in Java. The use of different languages in design and implementation is an advantage as it makes the distinction between these two stages very clear. We give examples showing that SML designs allow us to develop and compare OO implementations with different class structures for the same programming problem. A discussion of this kind is not supported in traditional $O O$ methodology. The program design in SML has also shown to be useful for the students when documenting the program.
\end{abstract}

Keywords - Functional Design, Object-Oriented Programs, Program Design, Implementation

\section{Introduction}

Teaching Java in an introductory level is a great challenge if you expect the students to do more than just play with GUI's and other parts of the technology. Choosing a good program structure (classes, objects and methods) for a given problem is quite difficult and there is not much help to get in a standard Java textbook. In a problem to be solved by the students you will hence have to add hints on the program structure unless the problem is so simple that the structure to be used is obvious. As a result, the students will learn something about Java, but they do not learn how to write a program. This paper discusses a different approach, which has been used for some years now, where we teach useful guidelines on how to structure a Java program by solving a given programming problem.

In the Informatics programme at the Technical University of Denmark, the first programming course is in functional programming using the SML programming language [3]. In this course, emphasis is put on structured data such as records, lists, disjoint unions, trees, sets and tables and their use in different kinds of applications. Furthermore, the rich type system of SML is used to express a program design in a succinct manner, where type expressions are used for data modelling and function types are used to specify the parameters and the results of the main tasks of the program.

After learning functional programming (in SML) the students are introduced to Object-Oriented $(\mathrm{OO})$ programming

1 Informatics and Mathematical Modelling, Technical University of Denmark, DK-2800 Lyngby, \{jtk,mrh,hsr\}@imm.dtu.dk (in Java). A major challenge of this course plan is to teach OO programming in a way that allows the students to take advantage of skills obtained in the preceding functional programming course. This is done by using program designs expressed in SML as the basis for Java implementations.

Our experience is that this gives a significant improvement in the quality of the produced Java code as well as the program documentation. The reason is, we believe, that making an SML design forces the students to clarify important concepts in the problem instead of rushing into implementation. This clarification of important concepts is of great value for implementation as well as for documentation.

An "SML design" in the sense of our course is an SML signature. From a methodology point of view, it covers the following decisions about the program:

- Concepts and terminology: names for important data and functions.

- Data: the structure of important data, expressed in terms of SML types of the data.

- Functions: the parameters and the results for important functions, expressed in terms of SML types of the functions.

The SML design helps the student in designing the overall structure of the program. It is our experience that this is the difficult part in developing a program - to complete the code is much easier. In doing so one must, however, consult the original problem statement to find out what each function "should do" as this information is not included into the SML design.

The SML design can be extended to cover also the dialogue with the user. This extension of the design describes the structure of the input and output data and the sequencing of inputs and outputs. We will, however, not pursue this part of the design in the present paper.

The transition from an SML design to the class structure of a Java program is comprised of the following decisions:

- identifying and naming the classes of the program,

- allocating each data item of the SML design to a specific class, and

- allocating each function of the SML design to a specific class.

These decisions fix the class structure of the program, so the code can now be filled in. In addition, the approach provides the basis for comparing and assessing possible $O O$ structures corresponding to the SML design.

In the next section we give an example-based introduction to SML designs and possible ways of deriving Java programs. Section III contains an example of symbolic differentiation 
Session T1D

involving expression trees. Section IV discusses the experience we have had with student's projects using SML designs as a basis for implementing as well as documenting the Java program. Finally, section $\mathrm{V}$ discusses a tool supporting the transition from SML designs to Java (skeleton) programs.

\section{Example}

In this section we present and discuss two different SML designs and their Java implementations. The presentation will be based on the following example:

A bank keeps track of the customer's bank accounts by a register which associates the balances of accounts with the names of customers. Furthermore, there are operations to create an empty register containing no accounts, insert a customer with a given balance into a register, to deduct an amount of money from a customer's account in a register, and to delete a customer from a register.

\section{Version 1: Focus on the structure of data}

In the following design of bank accounts with operations, we will focus on the structure of data. Important entities in the problem formulation, i.e., register, name, balance and amount are named and typed. Furthermore, types are provided for values and for functions, i.e., for empty register and for insert, deduct and delete:

type register

type name $=$ string

type balance $=$ int

type amount $=$ int

empty : register

insert: register * name * balance -> register

deduct: register * name * amount $\rightarrow$ register

delete: register * name $\rightarrow$ register

This is an example of an SML signature (in a slightly relaxed syntax) that expresses the SML design of the program.

Note that the use of SML signatures supports a succinct documentation of the program design. It gives a clear overview of important concepts, and we just need to add a short, textual explanation to the SML types of the functions. For example, the explanation for the deduction operation is: "The value of the expression deduct $(r e g, n, a)$ is the register obtained from reg by deducting $a$ dollars from person $n$ 's account".

We will assume that the old register needs not be retained when a new register has been computed. This is actually a property of the user interface which is not considered here. It is important to note that there are many Java implementations of this design.

An implementation focussing on the register

One observation of the design is that the functions take a register as part of their argument, and they give a new register as their result. Because we have assumed that the old register is not needed any more, it is natural to implement the design with a Java class Regis.ter containing a private data field to hold the current value of the register and methods for each of the specified functions.

This leads to the following Java skeleton:

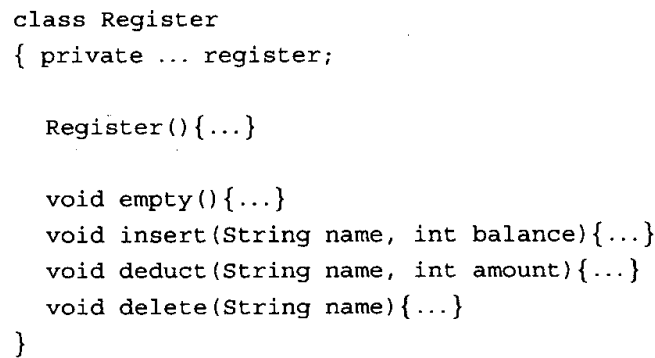

Note:

1. It is not decided how the register is represented, just that it is a private, mutable object, accessible by the methods of the class.

2. The program skeleton of each method is systematically derived from the corresponding function type.

3. The constructor Register and the method empty have the same functionality. Thus, the method empty is not needed in the final implementation.

The above sketch of a Java implementation has a drawback. It is not easy to extend. For example, if a new type of account is introduced for special customers, then one has to modify the above code and recompile the whole system. Similarly, a recompilation is needed if the representation of the register is changed for some reason. As a result, Java programs are usually written in a different way, as shown in Section B below.

\section{An implementation focussing on the customer}

In analysing the function types, one observes that the methods insert, deduct and delete could be hosted either in a register class or in a customer class. Customers are given by their names in the function types.

The methods in the Customer class then have a register as a parameter and name as a private data field. Details are left to the reader. Hence, this simple example shows that many implementation decisions have to be made in designing an objected-oriented implementation, e.g., which data should be arguments to methods and which should be private data fields of the objects.

\section{Version 2: Focus on a Register Interface}

In the design below, we declare an SML datatype command that describes the different operations and their parameters. We then only need a single function eval to execute any operation. The types for register, name, balance and amount are as before:

October 10-13, 2001 Reno, NV

31st ASEE/IEEE Frontiers in Education Conference

T1D-16 


\section{Session T1D}

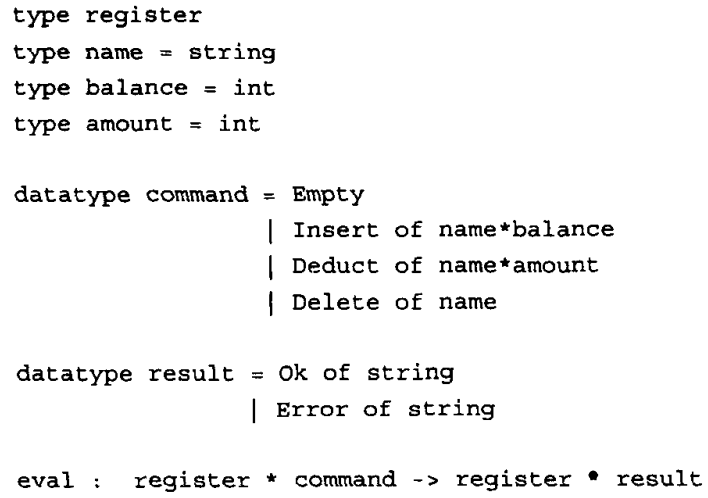

The different operations on the register are enumerated in the declaration of command, e.g., an insert operation is given by a name and a balance. Similarly, in the declaration of result, there is an enumeration of the different kinds of results of evaluating commands. The datatypes command and result are examples of disjoint unions. The register interface is comprised of commands, results and the evaluation function.

When we aim to implement a Java program that is easy to extend, e.g., with new kinds of accounts, there are two possibilities:

1. Operations on accounts are implemented by inheritance from a given class, e.g., a register class. New operations are then implemented and compiled separately, and this does not affect the existing implementation of the old operations on accounts.

2. Operations on accounts are described by a Java interface. Each command is obtained as an implementation of that interface. Hence, each command gets its own class and recompilation of old commands is not necessary when implementing new commands.

\section{An Implementation using Inheritance}

Looking at the above SML design, with an eye to achieving an easily extensible Java implementation, we observe that:

1. The introduction of a new command, for a new kind of account, must be accompanied with an evaluation function for that new command.

2. When updating a register, we assume as before, that the old register is not needed any more. As a result, we can make a class Register as before, with a private data field containing the current register.

3. The Java method eval can be hosted in the Register class, as the evaluation function takes the current register as part of its argument and gives a new register as part of its value.

A corresponding Java skeleton is:

class Register

\{ private static ... register;

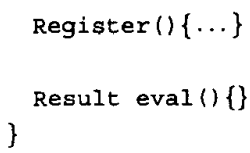

The implementation of the various commands is now obtained by extension of the Register class. In each case, one must give a constructor and an evaluation function for the command.

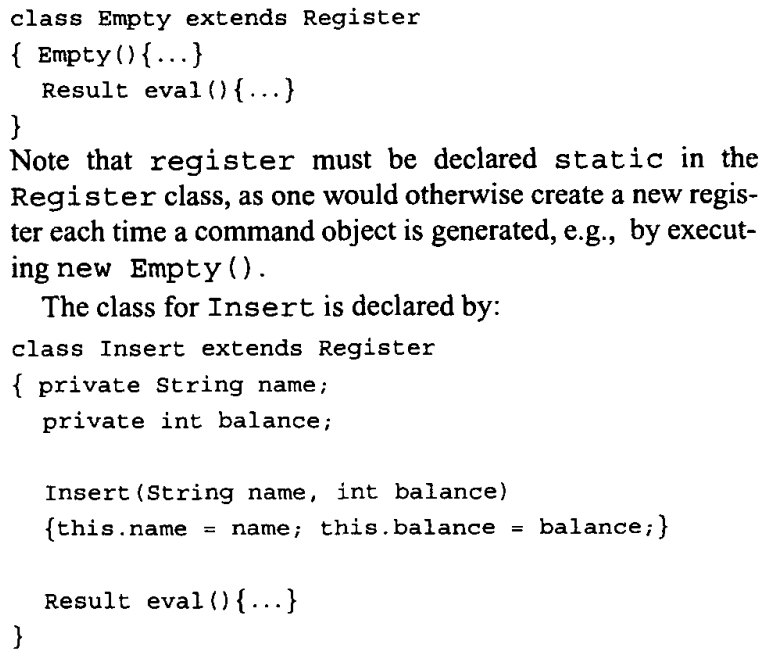
Register class, as one would otherwise create a new register each time a command object is generated, e.g., by executing new Empty ().

The class for Insert is declared by:

class Insert extends Register

\{ private string name;

private int balance;

Insert (String name, int balance)

\{this. name $=$ name; this. balance $=$ balance; $\}$

Result eval() $\{\ldots\}$

\}

The above declaration of the Java constructor Insert corresponds to the part: Insert of name*balance of the SML datatype declaration of command, which expresses that an insert command is given by: Insert $(n, b)$, where $n$ is a string (the name) and $b$ is an integer (the balance).

The remaining commands are implemented similarly:

class Deduct extends Register

$\{$ private string name;

private int amount;

Deduct (String name, int amount)

\{this.name $=$ name; this. amount $=$ amount $;\}$

Result eval () $\{\ldots\}$

\}

class Delete extends Register

$\{$ private string name;

Delete (String name) $\left\{\right.$ this name $=$ name $\left._{i}\right\}$

Result eval ( $)\{\ldots\}$

\}

It remains to give a Java implementation of the result datatype in the SML design expressing the possible results as the disjoint union of Ok-messages and Error-messages. We shall implement such disjoint unions by implementing an 


\section{Session T1D}

mand and result) from the SML design are represented the same way in the Java skeleton. Furthermore, it is much more natural that the evaluation function is associated with command classes, rather than with the Register class as it was the case in the previous implementation.

\section{Symbolic Differentiation}

This section shows how to obtain a Java program from an SML design containing a recursive datatype, using the derivation methods described in Section B.2. The example is that of symbolic differentiation, where expression trees are defined recursively, and where we use $D$ to denote the differentiation function:

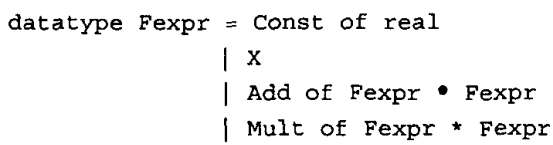

SML-values of the type Fexpr are expression trees like:

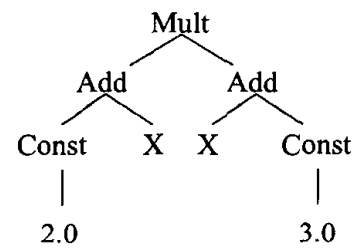

A tree representing $f(x)=(2+x) \cdot(x+3)$.

The abstract class for Fexpr is declared by:

interface Fexpr

\{

Fexpr $D()$

String tostring();

\}

where the interface contains the method D, as Fexpr is the argument type of the function $D$.

The Java skeletons for the various expression trees are:

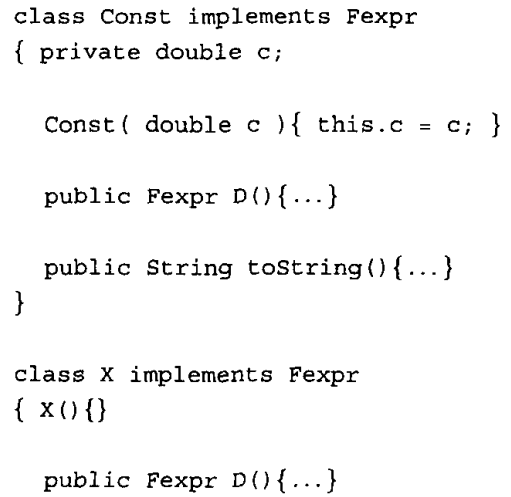

0-7803-6669-7/01/\$10.00 (c)2001 IEEE

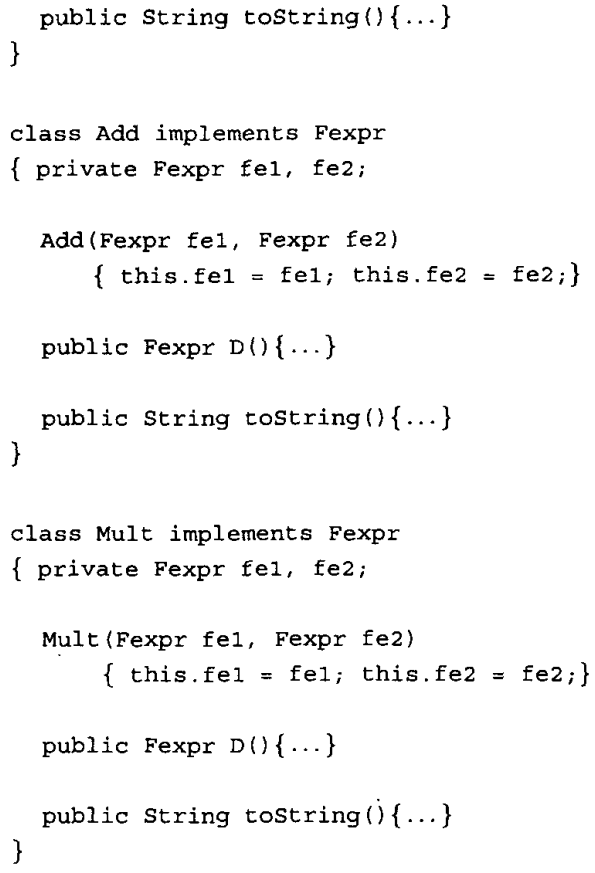

Having this skeleton, it is easy to fill out the remaining parts to get a complete program. We only give the remaining code for the Mult class:

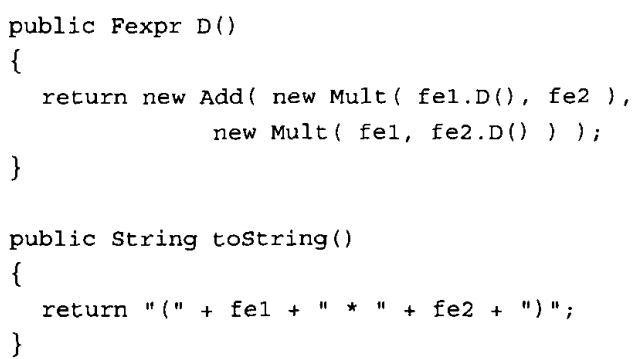

Note that only a few lines of code need to be added and that the declaration of the method $\mathrm{D}$ corresponds to the product rule for differentiation.

Symbolic differentiation is a complicated programming problem for second semester students in a Java course. Our experience is that many students are not able to solve it at all, while others come up with Java programs with a bad structure, i.e., programs that are hard to understand, even for the programmer.

Our approach gives an awareness on how to choose a class structure to get a proper Java implementation. This example shows that a method relating SML designs to Java skeletons helps students solve problems that they were not be able to solve properly otherwise.

October 10-13, 2001 Reno, NV

\section{1st ASEE/IEEE Frontiers in Education Conference}

T1D-19 
Session T1D

\section{Experience}

The students in our second semester Java course are evaluated based on reports documenting the solution to a software engineering problem. Previous experience with these reports has shown that the students had severe difficulties making a good object-oriented program as well as a good documentation of the program. Some years ago, the form of these projects has changed to require that students document the design using an SML design as described above. This has led to a significant improvement of both the implementation and the documentation.

The reason for these improvements are, we believe, that the SML design supports an abstract view of the system, where decisions about implementation details are postponed. In the example section, we saw how much freedom the SML design leaves in making an object-oriented representation of the account system. It also provides a useful frame of reference when comparing different object-oriented implementations. Furthermore, the abstract view of the SML design is a much better starting point for the documentation of the program, as it is not cluttered by implementation details. This positive experience with using SML designs, even when an object-oriented implementation was the goal, has led to focus on teaching systematic derivations of Java skeleton programs from SML designs.

\section{Automating the Derivations}

A prototype of a tool that can automatically generate Java skeleton programs from SML designs has now been constructed. Decisions about the OO structure appear as annotations to the SML design. A big advantage of the tool is that it generates a skeleton program which fits the chosen design, so that the programmer can begin filling in the implementation details of the specified entities. We saw in the section on symbolic differentiation, that only a few well-understood lines of code had to be added to the skeleton. Having a tool allows students to experiment with their design and the generated skeletons of the implementations. In the next semester, students will experiment with the tool.

\section{Acknowledgements}

We are grateful to the anonymous referees and Ann Quiroz Gates for very useful comments.

\section{References}

[1] Deimel, L.E. (Ed.), Software Engineering Education, LNCS, vol. 423, Springer-Verlag, 1990.

[2] Gamma, E., Helm, R., Johnson, R., Vlissides, J., Design Patterns, Addison-Wesley, 1995.

[3] Hansen, M.R., Kristensen, J.T., Rischel, H., A TheoryBased Introductory Programming Course, Frontiers in Education (FIE'99), Volume 1, pages $11 \mathrm{~b} 4-25$ to $11 \mathrm{~b} 4-30$, IEEE 1999.

0-7803-6669-7/01/\$10.00 C2001 IEEE
[4] Hansen, M.R., Rischel, H., Introduction to Programming using SML, Addison-Wesley, 1999.

[5] Sestoft, P., Kristensen, J.T., Ravn, A.P., Rischel, H., From Functional to Imperative Programming, Les languages applicatifs dans l'enseignement de l'informatique, pp. 26-33, Actes des 2èmes journées de travail, IFSIC-IRISA, Rennes 1993.

[6] Tucker, A.B. (Ed.), Computing Curricula 1991, Communications of the $A C M, 34$, June, pp. 69-84, 1991.

31st ASEE/IEEE Frontiers in Education Conference

October 10-13, 2001 Reno, NV

T1D-20 